\title{
Introducing a framework for process quality in National Statistical Institutes
}

\author{
Giovanna Brancato*, Francesco D’Assisi Barbalace, Marina Signore and Giorgia Simeoni \\ Italian National Statistical Institute, Rome, Italy
}

\begin{abstract}
The modernization programs that are being introduced in many National Statistical Institutes impose them to put at the heart of their concerns the assessment of performance and efficiency of statistical and organizational processes. So far, the international community attention has been more focused towards the quality of statistics, for which a highly structured and developed framework exists, supported by quality measures and proxy quality indicators. The Statistical Quality Control theory has acknowledged the importance of the processes, but still the attention is focused on statistical process quality improvement as a way to increase output quality. This paper is aimed at setting the building blocks for the development of a structured framework for the process quality itself. Processes are first defined and classified according to their different nature, e.g. statistical vs. organizational ones, taking into account both the management control literature and the UNECE Generic Activity Model for Statistical Organizations (GAMSO). Then, a set of quality characteristics applicable to the statistical processes and sub-processes and to some organizational processes is introduced. They are the pillars for the further definition of measures to monitor quality and performance and to assess them against given targets.
\end{abstract}

Keywords: Process quality characteristics, management process, business process

\section{Introduction}

The European Statistical System (ESS) can rely on a consolidated quality framework for assuring high quality statistics. The framework was developed over the last decades to respond to the requirements of the stakeholders and users of official statistics.

The European Statistics Code of Practice (ES CoP) [7] represents a cornerstone for quality activities of the European National Statistical Institutes (NSIs) and the other Statistical Authorities. As known, it encompasses the following areas: $i$ ) institutional environment, ii) statistical processes and iii) statistical products. Within each area principles to be fulfilled are stated. The ESS Quality Assurance Framework (QAF) [5] suggests methods and tools both at institutional level (meaning the entire organization) and at

${ }^{*}$ Corresponding author: Giovanna Brancato, Italian National Statistical Institute, Istat Via Cesare Balbo, 16, 00184 Rome, Italy. Tel. +3906 4673 3810; E-mail: brancato@ istat.it. process/product level in order to support the implementation of the ES CoP and its principles.

Nevertheless, the European quality framework is more developed with regard to product quality (i.e. the outputs of statistical production) than for process quality (i.e. the way by which outputs or products are produced). As a matter of fact, Eurostat quality dimensions clearly define the requirements for product quality that have to be satisfied in order to meet users' needs [7]. Furthermore, a set of standard Quality and Performance Indicators have been endorsed in the ESS in order to assess and communicate product quality to users [6].

Concerning statistical process quality, the ES CoP addresses the need that they are based on "sound methodologies", adopt "appropriate statistical procedures", place "non-excessive burden on respondents" and are conducted in a "cost-effective" way.

In 2001, the Leadership Expert Group (Leg) on Quality, for the first time acknowledged the importance of a Total Quality Management approach based on continuous quality improvement. The Leg on Qual- 
ity defined some characteristics that the statistical process should follow and recommended to measure key process variables in order to improve statistical processes [10].

Recently, the attention to process-oriented approaches and the focus to organizational issues have increased [4]. Indeed, in May 2014, the European Statistical System has adopted a common strategic response to the challenges that official statistics is facing, referred as ESS Vision2020 (http://ec.europa.eu/eurostat /web/ess/about-us/ess-vision-2020). One of the key areas of the ESS Vision2020 is the promotion of the efficiency in production processes. The importance to consider the other processes that contribute to the production of statistical outputs, besides the statistical ones, has also been recognized at international level [14]. Even though statistical processes have a direct impact on the outputs thus affecting their quality characteristics, it is true that the statistical production occurs in a wider context where other processes such as administrative, IT, knowledge management processes, are becoming more and more influential.

The modernization programs that are underway in many National Statistical Offices require the rethinking of the organization, the processes and the products and in particular the improvement of organizational efficiency [15].

Given all the above considerations, it is time now to focus on requirements and standard measures specifically designed to measure the process quality in itself.

This paper provides some inputs for the development of a structured framework for process quality. The aim is twofold: $i$ ) to define process quality requirements starting from the work done by the Leg on Quality as a first step to develop appropriate quality measures for processes and $i$ ) to extend the quality framework to other processes beyond statistical ones, recognizing the growing importance of such processes in the modernization of official statistics.

\section{Types of processes and process quality}

A process can be defined as "sequence of interdependent and linked procedures which, at every stage, consume one or more resources (employee time, energy, machines, money) to convert inputs (data, material, parts, etc.) into outputs. These outputs then serve as inputs for the next stage until a known goal or end result is reached". ${ }^{1}$ The process operates in a regulated

\footnotetext{
${ }^{1}$ http://www.businessdictionary.com/definition/process.html
}

environment. Essential factors of a "generic" process are then input(s), output(s) and a set of steps or activities that transform inputs into outputs.

A process classification widely used in the management control [2] identifies: i) Strategic processes; ii) Management processes; iii) Operational processes. They include the activities of strategic planning, management control and operational control, respectively. Strategic processes are those aimed at medium-long term planning of the organization. Management processes are those that support the translation of the medium-long terms objectives in the short term (annual) programs, and permit the control of the fulfilment of the objectives. Their clients are internal to the organization. Operational processes are those concurring to the achievement of the objectives of the organization, and respond to clients' needs "external" to the institution.

In other words operational processes correspond to Business processes. Nowadays, this terminology is commonly used also in the statistical context to refer to statistical processes.

Process quality has been widely studied in manufacturing, where its relevance has been recognized decades ago and methodologies for Statistical Quality Control (SQC) of industrial processes are well developed and currently used [9]. The underlying hypothesis is that improving process quality leads to better quality outputs. Indeed, SQC techniques such as Pareto diagrams or control charts, consist in monitoring key variables that, by definition, represent the quality of the output, while the process is ongoing and, if needed, appropriately act on the process to have an improving effect on the output. Thus, the focus is always on the output quality and the process is considered as a means to obtain the output. Product should meet specifications, any variation from specifications can be due to common (expected and unavoidable) causes or special (extra and unexpected) causes acting on the process. The process should be "stable", i.e. all special causes are removed, and "capable", i.e. the specifications should be met [11].

Use of SQC methods have been recommended in official statistics and examples of the application of the SQC in official statistics concern: the use of flow charts to outline the strategy in questionnaire design and testing; the control charts to monitor the performance of the interviewers during the survey data collection [1]; the use of Pareto analysis to improve the coding process [3].

Concerning the methods that are suggested in the QAF [5] for the principles of the statistical processes' 
area, at the institutional level they include the existence of an organizational structure which provides standards for methods and instruments and is in charge of the monitoring and assessment of their application. At the product/process level, methods to guarantee compliance with the principles are suggested with respect to the main phases of the statistical process: questionnaire design, sampling design, data collection and interviewers training, editing and imputation, estimation. In addition, a specific attention is devoted to the staff management (recruitment, training, motivation) and to the scientific cooperation with the academic world and with other NSIs. Methods to improve the use of administrative data for statistical purposes and to reduce the respondents' burden are also included. Finally, with respect to the principle on cost effectiveness, methods for a more efficient use of the resources are highlighted.

As it has been underlined in this section, the available methods oriented to process quality do not involve the improvement of quality characteristics of the processes themselves, that is indeed the approach introduced in this paper.

\section{Proposal for a framework on process quality}

\subsection{Scope of the framework}

When considering the statistical production, in line with the general definition, business process definition as defined in the Generic Statistical Information Model - GSIM [13] is "[t]he set of Process Steps to perform one or more Business Functions to deliver a Statistical Program Cycle or Statistical Support Program”. In addition, GSIM identifies support processes (Statistical Support Program) that can be directly serviceable to the statistical production or more crosssectional in nature, such as staff training.

A comprehensive list of the different activities in a statistical organization is reported in the Generic Activity Model for Statistical Organizations - GAM SO [14], and summarized in Table 1. As it can be observed, GAMSO approach is not distant from the classification introduced in Section 2, where the subactivities under the Strategy and Leadership area are Strategic Processes, those in the Capability Management and Corporate support are Management Processes and the Production activities are, obviously, the Business Processes whose description is provided by the Generic Statistical Business Process Model - GS BPM [12].
The GSBPM was developed some years before GAMSO and it is becoming the main reference model for describing "the set of business processes needed to produce official statistics". It provides "a template for process documentation, for harmonizing statistical computing infrastructures", and "a framework for process quality assessment and improvement" [12].

One of the goals of GAMSO is to extend and complement the GSBPM "by adding additional activities needed to support statistical production" [14]. These activities are important in the modernization of official statistics since "statistical production occurs within a broader context of corporate strategies, capabilities and support" [14].

The Strategy and leadership area has a straightforward correspondence to the Strategic Processes as described in Section 2. The Capability management and corporate support areas match with the Management Processes that aim at different purposes. The Capability management concerns the planning, development, monitoring and supporting of the implementation of the "capabilities that underpin an organization's ability to conduct its business" [14]. The Corporate support encompasses "the cross-cutting" functions required by the organization to deliver its work program efficiently and effectively" [14] covering activities such as Business and performance, finances, human resources, IT, methodology, information and knowledge, consumers, data suppliers, physical spaces, and last but not least quality.

Thus, GAMSO distinguishes the area where capabilities, e.g. re-use or sharing of infrastructures and technologies, are planned and developed from the area where its corporate implementation is accomplished (i.e. corporate support area).

In an holistic perspective each activity (and related processes) is relevant for quality, thus it can be monitored and assessed according to a process quality perspective. This idea is not new for business processes, even though the focus was more on the output quality than on the process itself (see Section 1).

In the next Section some process quality characteristics are proposed. They are applicable to the business processes and the subset of management processes represented by the GAMSO's activities in the Corporate support area.

\subsection{Process quality characteristics}

"The product is generated by an underlying process. It is unlikely that the product will have good quality if 
Table 1

Strategic, management and business process mapped in GAMSO

\begin{tabular}{ll}
\hline GAMSO areas and top and second main hierarchical levels & Type of processes \\
\hline Strategy \& leadership (Define vision, Govern \& Lead, Manage strategic collaborations) & Strategic \\
Capability management (Plan, develop, monitor and support implementation of capabilities) & Management \\
Corporate support (Manage business performance, finances, HR, IT, methodology, knowledge, suppliers, physical & \\
spaces, quality) & Business \\
Production (Statistical business processes) & \\
\hline
\end{tabular}

Table 2

Eight elements of St. Thomas Aquinas

\begin{tabular}{ll}
\hline Element & Meaning \\
\hline "Quis" (Who) & Who is responsible and who carries out the process/phase/sub-process \\
"Quid" (What) & What is done in each process/phase/sub-process \\
"Quando" (When) & Each process/phase/sub-process has a starting and ending dates (time length) \\
"Ubi" (Where) & Where the process/phase/sub-process is carried out, e.g. internally or externally; centralized or de- \\
& centralized \\
"Cur" (Why) & Aim of the process/phase/sub-process \\
"Quantum" (How many, how much) & Volume of input and output for each process/phase/sub-process \\
"Quomodo" (How) & Methodologies, techniques, procedures, software and tools (e.g. protocols, agreements, contracts, \\
& ...) for each process/phase/sub-process \\
\hline
\end{tabular}

the underlying process is not up to par" [10]. Analogously to the quality framework for product quality, we can also define quality characteristics for the process. As already mentioned, several characteristics and requirements for process quality have already been identified by the Leadership Group on Quality [10]. ${ }^{2}$

In this section, we suggest an extension of the quality requirements that a statistical business process should satisfy in order to ensure an adequate level of quality, starting from those already proposed by the Leg on Quality [10]. Some quality requirements are gaining importance in the context of the modernization of official statistics and in the application of the international standards recently developed (as GAMSO and GSBPM).

The following set of requirements is proposed for business and corporate support processes of a statistical organization: identified, transparent, reproducible, reliable and robust, effective, efficient, controllable, flexible, integrated and secure. These requirements may be related to the process as a whole or to its components, i.e. phases and sub-processes following GSBPM terminology.

Identified. The process/phase/sub-process is well defined and mapped to a standard model or tool. In order to be identified, a process should be specified according to the well-known rule of $5 \mathrm{~W}$ (who, what, when, where, why) or even to the 8 elements of St.

\footnotetext{
${ }^{2}$ The aims of process quality are to gain efficiency, effectiveness, robustness, flexibility, transparency, and integration [10].
}

Thomas Aquinas (quis, quid, when, ubi, cur, quantum, quomodo, quibus auxiliis) from which the rule of $5 \mathrm{~W}$ derives, as specified in Table 2 .

Transparent. The process/phase/sub-process is documented in a clear, complete, accurate and updated way and the documentation is easily accessible to users and stakeholders.

Reproducible. The process/phase/sub-process produces the same output if replicated on the same input.

Reliable and robust. The process/phase/sub-process is reliable if its correct functioning is guaranteed. For example reliability could be measured as the probability that an IT system does not break down. The process/phase/sub-process is robust if it achieves results against unexpected situations.

Effective. The process/phase/sub-process is successful in delivering the desired outcomes.

Efficient. The process/phase/sub-phase produces the expected outputs cost-effectively. Gains in efficiency are obtained when a process delivers the expected results with the same quality but with less resources (in terms of personnel, costs or both).

Controllable. The process/phase/sub-process is monitored and assessed against targets.

Flexible. The process/phase/sub-process is readily adaptable to changing needs and demands.

Integrated. The process/phases/sub-process can be easily combined with other processes/phases/subprocesses.

Secure. The risks associated to process/phase/subprocess are analysed and prevented. For business pro- 
cesses risk analysis should also refer to possible loss of data (input and output). Data confidentiality should be guaranteed in each process step.

It is worthy to notice that a process which is identified, transparent and reproducible in all its phases/subprocesses allows for the traceability of the data along the whole statistical business process.

The proposed process quality characteristics seem to apply to any kind of process/phase/sub-process within a statistical business and a corporate support process. Particularly, they have been tested on to two subprocesses: $i$ ) the selection of an outsourcing company for the Computer Assisted Telephone Interviewing and ii) the coding of textual variables procedure. The first sub-process is a corporate support process, the second one is part of the business process. Most process quality characteristics are straightforward for both applications, for example: identified, transparent, reproducible, effective, efficient, controllable and flexible. Other characteristics, such as reliable and robust may as well apply, but may be more relevant for those subprocesses performed via IT.

Finally, the relevance of some process quality characteristics may vary depending on the some organizational factors. For example, the characteristic "integrated" can become highly relevant in the context of the modernization of statistics, where some services are centralized.

\section{Concluding remarks}

In this paper, starting from the work laid down by the Leg on Quality [10], a set of process quality characteristics has been proposed. They are the pillars for developing a structured framework for quality monitoring and assessment, encompassing proper measurements and evaluation methods. Given the growing importance of the organizational activities, well acknowledged in GAMSO [14], the framework has been extended to corporate support processes not yet considered from a quality perspective, as far as we know.

Similarly to what happens for statistics quality dimensions, it is expected that quantitative measures will be more easily available only for a subset of characteristics, and not for all of them. In addition, the measures for some characteristics will be the same independently if referred to statistical business processes or to corporate support ones. A trivial example is the duration of the process/phase/sub-process. In other cases, the measures might be more dependent on the nature of the process/phase/sub-process.
As a starting point, some methods reported in Aitken et al. [1], the handbook on process variables developed on the basis of Leg on Quality recommendation n.2. ${ }^{3}$, can be borrowed in order to provide the process quality characteristics introduced in this paper with qualitative or quantitative assessment measurements.

As a final goal every organization should be able to introduce and maintain a quality management based on measurements and assessment of tailored indicators for both business and corporate support processes in a holistic approach.

\section{References}

[1] A. Aitken, J. Hörngren, N. Jones, D. Lewis and M.J. Zilhão, Handbook on improving quality by analysis of process variables; 2004. Available from: http://ec.europa.eu/eurostat/docu ments/64157/4373903/06-Handbook-on-improving-qualityby-analysis-of-process-variables.pdf.

[2] R.N. Anthony, Planning and Control: a Framework for Analysis. Cambridge MA. Harvard University Press, 1965.

[3] P.P. Biemer and R.A. Caspar, Continuous Quality Improvement for Survey Operations: Some General Principles and Applications, Journal of Official Statistics 10(3) (1994), 307326.

[4] CES. In depth review of process-oriented approach to statistical production. Note by Turkish Statistical Institute. Conference of European Statisticians. United Nations, Geneva, Switzerland, 19-20 February 2015.

[5] Eurostat. Quality Assurance Framework of the European Statistical System. V1.2; 2015. Available from: http://ec.europa .eu/eurostat/documents/64157/4392716/ESS-QAF-V1-2final .pdf/bbf5970c-1adf-46c8-afc3-58ce177a0646.

[6] Eurostat. ESS Guidelines for the implementation of the ESS Quality and Performance Indicators (QPI); 2014. Available from: http://ec.europa.eu/eurostat/documents/64157/437 3903/02-ESS-Quality-and-performance-Indicators-2014.pdf.

[7] Eurostat. European Statistics Code of Practice; 2011. Available from: http://epp.eurostat.ec.europa.eu/cache/ITY_OFF PUB/KS-32-11-955/EN/KS-32-11-955-EN.PDF.

[8] G.A. Gorry, M. Scott and S. Michael, A framework for management information systems. Sloan Management Review (Massachusetts Institute of Technology), 1971.

[9] E.L. Grant and R.S. Leavenworth, Statistical quality control. 7th edition, New York, McGraw-Hill, 1996.

[10] L. Lyberg, M. Bergdahl, M. Blanc et al., Summary Report from the Leadership Group (LEG) on Quality; 2001. Available from: http://www.istat.it/it/files/2011/11/LEG-onquality.pdf.

[11] T.P. Ryan, Statistical methods for quality improvement. Second edition. New York: Wiley, 2000.

[12] United Nations Economic Commission for Europe (UNECE). GSBPM, Generic Statistical Business Process Model v5.0; 2013. Available from: http://www1.unece.org/stat/platform/ display/GSBPM/GSBPM+v5.0.

\footnotetext{
3 "Process measurements are vital for all improvement work. A handbook on the identification of key process variables, their measurement, and measurement analysis should be developed." [10]
} 
[13] United Nations Economic Commission for Europe (UNECE). Generic Statistical Information Model (GSIM): Communication Paper for a General Statistical Audience (Version 1.1, December 2013); 2013. Available from: http://www1.unece.org/ stat/platform/display/gsim/GSIM+Communication+Paper.

[14] United Nations Economic Commission for Europe (UNECE). GAMSO, Generic Activity Model for Statistical Organisa- tions. Version 1.0: 1 March 2015; 2015. Available from: http:// www1.unece.org/stat/platform/display/GAMSO/GAMSO+ v1.0.

[15] S. Vale, Modernisation of Official Statistics - An international perspective; 2015. 60th ISI World Statistics Congress, Rio de Janeiro, Brasil. 